[1] K. Clancey, Seminormal Operators, Lecture Notes in Math. 742, Springer, 1979.

2] R. L. Dobrushin and R. A. Minlos, Polynomials of linear random functions, Uspekhi Mat. Nauk 32 (2) (194) (1977), 67-122 (in Russian).

[3] A. V. Marchenk o, Selfadjoint differential operators with an infinite number of independent variables, Mat. Sb. 96 (2) (1975), 276-293 (in Russian).

[4] W. Mlak, Introduction to the Theory of Hilbert Spaces, PWN, Warszawa 1982 (in Polish).

[5] -, Operators induced by change of Gaussian variables, Ann. Polon. Math., to appear.

[6] A. Pokrzywa, On continuity of spectra in norm ideals, Linear Algebra Appl. 69 (1985), 121-130.

[7] M. Reed and B. Simon, Methods of Modern Mathematical Physics II: Fourier Analysis, Self-adjointness, Academic Press, New York 1975.

[8] M. Rosenblum, On a theorem of Fuglede and Putnam, J. London Math. Soc. 33 (1958), 376-377.

[9] J. K. Rudol, The spectrum of orthogonal sums of subnormal pairs, preprint, 1985.

[10] Yu. S. Samoilenko, Spectral Theory of Systems of Selfadjoint Operators, Naukova Dumka, Kiev 1984

[11] J. Stochel and F. Szafraniec, Bounded vectors and formally normal operators, Operator Theory: Adv. Appl. 11 (1983), 363-370.

[12] Y. Yamasaki, Kolmogorov's extension theorem for infinite measures, Publ. RIMS Kyoto Univ. 10 (1975), 381-411.

INSTYTUT MATEMATYCZNY POLSKIEJ AKADEMII NAUK

ODDZIAL W KRAKOWIE

INSTITUTE OF MATHEMATICS, POLISH ACADEMY OF SCIENCES

KRAKÖW BRANCH

Solskiego 30, 31-027 Kraków, Poland

\section{Groups of isometries on operator algebras}

by

\section{STEEN PEDERSEN (Aarhus)}

Abstract. Let $\varrho$ be a $C_{0}$-group of isometries on a unital $C^{*}$-algebra $A$. If $u(t)=\varrho(t) 1$ and $\alpha(t) a=u(t)^{*} \varrho(t) a$, then $\varrho(t) a=u(t) \alpha(t) a, \alpha$ is a $C_{0}$-group of $*$-automorphisms on $A$ and $u$ is a unitary 1-cocycle. We study this decomposition of $\varrho$; as a consequence we obtain a classification of the generators of $C_{0}$-groups of isometries on $A$.

Introduction. In [18] Kadison proved that an isometry of a unital $C^{*}$-algebra $A$ onto itself can be decomposed into a $C^{*}$-homomorphism followed by multiplication by a unitary. We study the consequences of applying this decomposition to a strongly continuous isometric representation $\varrho$ of a topological group on $A$. We prove that the $C^{*}$-homomorphic part of $\varrho$ is a strongly continuous group of $*$-automorphisms and that $\varrho$ is norm-continuous if $A$ is a von Neumann algebra. We establish conditions, global as well as local, which are satisfied by $\varrho$ if and only if it is a group of $*$-automorphisms.

Using perturbation theory for *-automorphism groups we prove that if $\varrho$ is a one-parameter group of isometries on $A$ with generator $\delta$, then there exist $(\gamma, v, h)$, where $\gamma$ is the generator of a one-parameter group of *-automorphisms on $A, v$ is a unitary in $A$ and $h$ is a selfadjoint element of $A$, such that $\mathscr{D}(\delta)=v^{*} \mathscr{D}(\gamma)$ and

$$
\delta(a)=v^{*} \gamma(v a)+i v^{*} h v a
$$

for $a$ in $\zeta(\delta)$. Using this we give local and global conditions equivalent to the fact that the unitary part of $\varrho$ is a group.

In the next part of the paper we specialize to the case where $\varrho$ is a one-parameter group. We observe that in some representations of $A, \varrho(t) a$ $=u(t) a v(t)$, where $u$ and $v$ are strongly continuous unitary groups. We study the generators of (semi-) groups of this form.

This study was motivated by applications to quantum mechanics (e.g. [15], [22], [25]) and partially inspired by the corresponding problems for a one-parameter semigroup on a Hilbert space if each element of the semigroup is polar decomposed [11], [12]. 
\$0. Notation. If $X$ is a Banach space, then $B(X)$ denotes the algebra of all bounded linear maps from $X$ into $X$. If $G$ is a topological space and $\varrho$ is a map from $G$ into $B(X)$, then $\varrho$ is strongly continuous if $g \rightarrow \varrho(g) f$ is a continuous map from $G$ into $X$ for each $f$ in $X$.

If $G$ is a group; then a map $\varrho$ from $G$ into $B(X)$ is called a representation of $G$ on $X$ if $\varrho(e)=1$ and $\varrho(g h)=\varrho(g) \varrho(h)$ for $g$ and $h$ in $G$, where $e$ is the unit in $G$ and 1 is the identity on $X$. A representation of the additive group of real numbers will sometimes be called a one-parameter group.

Let $\boldsymbol{R}_{+}$denote the set of nonnegative real numbers. A strongly (resp. weak ${ }^{*}$ ) continuous semigroup on $X$ (resp. $X^{*}$ ) is a map $\varrho$ from $\boldsymbol{R}_{+}$into $B(X)$ (resp. $B\left(X^{*}\right)$ ) such that $\varrho$ is strongly continuous, $\varrho(0)=1$ and $\varrho(s+t)$ $=\varrho(s) \varrho(t)$ for $s$ and $t$ in $\boldsymbol{R}_{+}$(resp. there exists a strongly continuous semigroup $\varrho_{*}$ on $X$ such that $\varrho(t)=\varrho_{*}(t)^{*}$ for $t$ in $\left.\boldsymbol{R}_{+}\right)$. If $\varrho$ is a strongly continuous semigroup, then the generator $\delta$ of $\varrho$ is defined by

$$
\delta(f)=\lim _{t \downarrow 0}(\varrho(t) f-f) / t,
$$

the domain $\mathscr{D}(\delta)$ of $\delta$ being the $f$ in $X$ where the limit exists. If $\varrho$ is weak* continuous, then the generator of $\varrho$ is the adjoint of the generator of $\varrho_{*}$. If $\varrho$ is a strongly (weak*) continuous semigroup with generator $\delta$, then we write $\varrho(t)=\exp (t \delta)$.

If $\varrho$ is a representation (resp. semigroup), then $\varrho$ is an isometric (contraction etc.) representation (resp. semigroup) if each $\varrho(g)$ is an isometry (contraction etc.).

Let $A$ be a $C^{*}$-algebra with unit 1 . Denote by $U(A)$ the unitary group in $A$. For $b$ in $A$ denote by $L(b)$ (resp. $R(b)$ ) the element in $B(A)$ defined by $L(b) a=b a$ (resp. $R(b) a=a b$ )

Fix $\alpha$ in $B(A) . \alpha$ is called a $C^{*}$-homomorphism if $\alpha(1)=1, \alpha\left(a^{*}\right)=\alpha(a)^{*}$ for $a$ in $A$, and $\alpha\left(a^{2}\right)=\alpha\left(a^{2}\right)$ for selfadjoint $a$ in $A$. $\alpha$ is a $(*-)$ homomorphism if $\left(\alpha\left(a^{*}\right)=\alpha(a)^{*}\right.$ and) $\alpha(a b)=\alpha(a) \alpha(b)$ for $a$ and $b$ in $A$. $\alpha$ is an antihomomorphism if $\alpha(a b)=\alpha(b) \alpha(a)$ for $a$ and $b$ in $A$.

Let $\delta$ be a linear (unbounded) map on $A$, with domain $\delta(\delta)$. Then $\delta$ is symmetric if $a \in \mathscr{D}(\delta)$ implies $a^{*} \in \mathscr{D}(\delta)$ and $\delta\left(a^{*}\right)=\delta(a)^{*}, \delta$ is a derivation if $a, b \in \mathscr{D}(\delta)$ imply $a b \in \mathscr{D}(\delta)$ and $\delta(a b)=\delta(a) b+a \delta(b)$. A symmetric derivation is called a $*$-derivation.

For background material on $C^{*}$-algebras, representations and semigroups, we refer the reader to [3], [8], [24] and [27].

$\S 1$. Groups on $C^{*}$-algebras. We introduce the polar decomposition of an isometric representation $\varrho$ of a group $G$ on a unital $C^{*}$-algebra $A$, and we investigate some of its basic properties.

The following result is an immediate consequence of [18, Theorem 7] and [26, Corollary 2].
THEOREM 1.1. Let $A$ be a unital $C^{*}$-algebra and let $\varrho$ be a bounded linear map on $A$. If $\varrho$ is surjective, then $\varrho$ is an isometry if and only if $\varrho(U(A)) \subseteq U(A)$. If $\varrho(U(A)) \subseteq U(A)$ then $\varrho=L(u) \alpha$, where $u$ is in $U(A)$ and $\alpha$ is a $C^{*}$-homomorphism; the decomposition is unique.

Definition 1.2. The decomposition $\varrho=L(u) \alpha$ in Theorem 1.1 will be called the polar decomposition of $\varrho$, because it is analogous to the polar decomposition of Hilbert space operators.

If $\varrho$ is a map from a set $G$ into $B(A)$ and $\varrho(g) U(A) \subseteq U(A)$ for each $g$ in $G$, then we define $u: G \rightarrow U(A)$ and $\alpha: G \rightarrow B(A)$ by the requirement that $\varrho(g)=L(u(g)) \alpha(g)$ is the polar decomposition of $\varrho(g)$ for each $g$ in $G$. We call $u$ (resp. $\alpha$ ) the unitary (resp. positive or $C^{*}$-homomorphic) part of $\varrho$, and the pair $(u, \alpha)$ is called the polar decomposition of $\varrho$. Note that $(u, \alpha)$ is determined by

$$
u(g)=\varrho(g) 1 \in U(A), \quad \alpha(g)=L\left(u(g)^{*}\right) \varrho(g) \in B(A)
$$

for $g$ in $G$. In the following two results we study the continuity properties of $u$ and $\alpha$.

Proposition 1.3. Let $G$ be a topological space and let $\varrho: G \rightarrow B(A)$ be strongly continuous. Assume that $\varrho(g) U(A) \subseteq U(A)$, and let $(u, \alpha)$ be the polar decomposition of $\varrho$. Then $g \rightarrow u(g)$ is a continuous function from $G$ into $A$, and $\alpha$ is strongly continuous.

Proof. $u(g)=\varrho(g) 1$ is continuous by assumption. Since

$$
\|\alpha(g) a-\alpha(h) a\| \leqslant\|u(g)-u(h)\|\|a\|+\|\varrho(g) a-\varrho(h) a\|
$$

for $g, h$ in $G$ and $a$ in $A$ we conclude that $\alpha$ is strongly continuous.

TheOREM 1.4. Let $G$ be a connected topological space, and let $\alpha$ be a strongly continuous map from $G$ into the surjective $C^{*}$-homomorphisms on a unital $C^{*}$-algebra $A$. If there exists $e$ in $G$ such that $\alpha(e)$ is a *-homomorphism, then $\alpha(g)$ is a *-homomorphism for each $g$ in $G$.

Proof. We only need to prove that $\alpha(g)$ is a homomorphism for each $g$ in $G$. Let $\pi$ be an irreducible representation of $A$, and let $G_{\mathrm{h}}(\pi)$ (resp. $G_{\text {ah }}(\pi)$ ) be the set of $g$ in $G$ for which $\pi \alpha(g)$ is a homomorphism (resp. antihomomorphism). By [19, Theorem 2.6], $G$ is the union of $G_{\mathrm{h}}(\pi)$ and $G_{\mathrm{ah}}(\pi)$. We will prove that $G=G_{\mathrm{h}}(\pi)$. It is easy to see that both $G_{\mathrm{h}}(\pi)$ and $G_{\mathrm{ah}}(\pi)$ are closed. If the intersection of $G_{\mathrm{h}}(\pi)$ and $G_{\mathrm{ah}}(\pi)$ is empty, then the proof is complete. Choose $g$ in the intersection of $G_{\mathrm{h}}(\pi)$ and $G_{\mathrm{ah}}(\pi)$. Since $\pi \alpha(g)$ maps $A$ onto $\pi(A)$ the existence of such a $g$ implies that $\pi(A)$ is abelian, hence $G$ $=G_{\mathrm{h}}(\pi)=G_{\mathrm{ah}}(\pi)$. Since the direct sum of all irreducible representations of $A$ is faithful, each $\alpha(g)$ is a homomorphism, and this completes the proof of the theorem. 
The following formula is the major tool used in the case where $\varrho$ is an isometric representation of a group.

LEMMA 1.5. Let $\varrho$ be an isometric representation of a group $G$ on a unital $C^{*}$-algebra $A$. If $(u, \alpha)$ is the polar decomposition of $\varrho$, then $u$ is a 1-cocycle w.r.t. $\alpha$, i.e.

$$
u(g h)=u(g) \alpha(g)(u(h))
$$

for $g$ and $h$ in $G$.

Proof. Since $\varrho$ is a representation,

$$
u(g h)=\varrho(g h) 1=\varrho(g)(\varrho(h) 1)=u(g) \alpha(g)(u(h))
$$

which proves the lemma.

The following theorem is the major consequence of the results obtained above.

THEOREM 1.6. Let $G$ be a connected topological group, $A$ a unital $C^{*}$. algebra and $\varrho$ a strongly continuous isometric representation of $G$ on $A$. Let $u(g)=\varrho(g) 1$ and $\alpha(g)=L\left(u(g)^{*}\right) \varrho(g)$. Then $\alpha$ is a strongly continuous representation of $G$ on $A$ by *-automorphisms.

Proof. By Proposition 1.3 and Theorem 1.4 it is enough to prove that $\alpha(g h)=\alpha(g) \alpha(h)$ for $g$ and $h$ in $G$. By. Lemma 1.5

$$
\begin{aligned}
u(g h) \alpha(g h) a & =\varrho(g h) a=\varrho(g)(\varrho(h) a)=u(g) \alpha(g)(u(h) \alpha(h) a) \\
& =u(g) \alpha(g)(u(h)) \alpha(g)(\alpha(h) a)=u(g h) \alpha(g)(\alpha(h) a)
\end{aligned}
$$

for $a$ in $A$. This proves the theorem.

COROLlary 1.7. Let $\varrho$ be a strongly continuous isometric representation of a connected topological group $G$ on a von Neumann algebra. If the topology on $G$ is metrizable, then $\varrho$ is norm-continuous, i.e. $\|\varrho(g)-1\| \rightarrow 0$ as $g \rightarrow e$.

Proof. $\alpha$ is norm-continuous by [10] or [20] hence $\varrho(g)=L(u(g)) \alpha(g)$ is norm-continuous by Proposition 1.3.

The case where the von Neumann algebra is all of $B(\mathscr{H})$ was considered in [2]. Note that if a topological group $G$ satisfies the first axiom of countability, then the topology on $G$ is metrizable [23].

Remark 1.8. We have the following converse of Theorem 1.6. Let $G$ be a group, $u$ a map from $G$ into $U(A)$ and $\alpha$ a representation of $G$ as *automorphisms of $A$. If $u$ is a 1-cocycle w.r.t. $\alpha$, then $\varrho(g)=L(u(g)) \alpha(g)$ defines an isometric representation of $G$ on $A$ with polar decomposition $(u, \alpha)$
$\S 2$. The unitary part. Let $G$ be a group and $\varrho$ an isometric representation of $G$ on a unital $C^{*}$-algebra $A$. In this section we give global conditions which make the unitary part of the polar decomposition $(u, \alpha)$ of $\varrho$ a representation of $G$.

The following result follows immediately from the cocycle property of $u$ (Lemma 1.5).

Proposition 2.1. Fix $g$ and $h$ in $G$. Then $u(g h)=u(g) u(h)$ if and only if $\alpha(g)(u(h))=u(h)$.

Proposition 2.2. If $G$ is a connected topological group and $\varrho$ is strongly continuous, then the following three conditions are equivalent:

(1) $u$ is a representation.

(2) The range of $u$ is a subset of the fixed point algebra for the action of $G$ on $A$ by $\alpha$.

(3) $L(u(g))$ and $\alpha(h)$ commute for-all $g$ and $h$ in $G$.

Proof. $(1) \Leftrightarrow(2)$ is a trivial consequence of Proposition 2.1. Since $\alpha(h)$ is a homomorphism (Theorem 1.4) we get

$$
\alpha(h) L(u(g)) a=\alpha(h)(u(g)) \alpha(h) a
$$

for $a$ in $A$. Using this it is easy to see that (2) and (3) are equivalent.

Definition 2.3. Let $\varrho \in B(A)$ with $\varrho U(A) \subseteq U(A)$ and let $\varrho=L(u) \alpha$ be the polar decomposition of $\varrho$. $\varrho$ is said to be quasi-normal if $L(u)$ and $\alpha$ commute.

Remark 2.4. The term "quasi-normal" is chosen because a linear map $H$ on a Hilbert space is quasi-normal if and only if $P U=U P$, where $H$ $=U P$ is the polar decomposition of $H$.

The following theorem is similar to [12, Theorem 2] (cf. also [11, Theorem 6]).

THEOREM 2.5. Let $G$ be either the group of real numbers or the group of complex numbers of modulus one. If $\varrho$ is a strongly continuous isometric representation of $G$ on a unital $C^{*}$-algebra, then the following two conditions are equivalent

(1) $\varrho(g)$ is quasi-normal for each $g$ in $G$.

(2) $u(g)=\varrho(g) 1$ is a representation of $G$

Proof. (2) $\Rightarrow(1)$ follows from Proposition 2.2.

$(1) \Rightarrow(2)$. First we consider the case where $G$ is the additive group of reals. Since $\varrho$ and $\alpha$ are representations we get

$$
L(u(n t)) \alpha(n t)=L(u(t))^{n} \alpha(n t)
$$


for $t$ in $G$ and $n=1,2,3, \ldots$; therefore

$$
u(n t)=u(t)^{n}
$$

for $t$ in $G$ and $n=1,2,3, \ldots$; in particular,

$$
u(p / q+r / s)=u(1 /(q s))^{p s+q r}=u(p / q) u(r / s)
$$

for $p, q, r, s=1,2,3, \ldots ;$ by continuity

$$
u(s+t)=u(s) u(t)
$$

for $s, t \geqslant 0$. Similarly one proves that $u(s+t)=u(s) u(t)$ for $s, t \leqslant 0$. By these equalities and the norm continuity of $u$

$$
\lim _{t \downarrow 0}(\varrho(t) 1-1) / t \quad \text { and } \quad \lim _{t \uparrow 0}(\varrho(t) 1-1) / t
$$

exist, hence $1 \in \mathscr{D}(\delta)$, where $\delta$ is the generator of $\varrho$ [3], [8]. We deduce that

$$
\lim _{t \downarrow 0}(u(t)-1) / t=\delta(1)=\lim _{t \uparrow 0}(u(t)-1) / t .
$$

Hence $u(t)=\exp (t \delta(1))$ for $t$ in $G$, in particular

$$
u(s+t)=u(s) u(t)
$$

for $s$ and $t$ in $G$.

If $G$ is the multiplicative group of complex numbers of modulus one, apply the result above to $t \rightarrow u\left(e^{i t}\right)$. This completes the proof.

Remark 2.6. If $T(t)$ is a strongly continuous one-parameter (semi-) group on a Hilbert space and $T(t)=U(t) P(t)$ is the polar decomposition of each $T(t)$, then [12] $U(t)$ is a (semi-) group if $P(t)$ is a (semi-) group.

Next we indicate how one may construct a strongly continuous oneparameter group $\varrho$ of isometries on a unital $C^{*}$-algebra $A$ such that the unitary part of $\varrho$ is not a representation. The construction is carried out in terms of the polar decomposition $(u, \alpha)$ of $\varrho$ (cf. Remark 1.8).

Fix a strongly continuous one-parameter group $\alpha$ of $*$-automorphisms on $A$. For each selfadjoint $h$ in $A$, the solution to

$$
\frac{d}{d s} u(s)=i u(s) \alpha(s) h, \quad u(0)=1
$$

is a norm-differentiable unitary 1-cocycle w.r.t. $\alpha$ [1, Theorem 2]. It is easy to see that $\alpha(t) u(s)=u(s)$ (all real $s$ and $t$ ) implies that $\alpha(t) H=h$ (all $t$ ); hence by Proposition 2.1, $u$ is a representation if and only if $\alpha(t) h=h$ for all $t$.

$\S 3$. Generator results. In this section, we obtain a characterization of the generators of isometric one-parameter groups on a unital $C^{*}$-algebra. Special attention is paid to the situation where the unitary part is differentiable. Further, quasi-normal groups are classified in terms of their generators.

Theorem 3.1. Let $A$ be a unital $C^{*}$-algebra and let $\delta$ be a linear map on $A$ with domain $\mathscr{D}(\delta) . \delta$ is the generator of a strongly continuous one-parameter group of isometries on $A$ if and only if there exists $a$ selfadjoint $h$ in $A$ and $a$ unitary $v$ in $A$ such that

$$
\gamma(a)=v \delta\left(v^{*} a\right)-i h a
$$

for a in $\mathscr{D}(\gamma)=v \mathscr{D}(\delta)$, is the generator of a strongly continuous one-parameter group of *-automorphisms on $A$.

Proof. Let $\varrho(t)=\exp (t \delta)$ be a group of isometries, and let $(u, \alpha)$ be the polar decomposition of $\varrho$. Since $u$ is a 1-cocycle w.r.t. $\alpha$, it follows from [6, Corollary 4.4] that there exists $v$ in $U(A)$ and a (norm-) differentiable 1 cocycle $w$ such that $u(t)=v^{*} w(t) \alpha(t)(v)$. By [1] and [6, Proposition 4.6], w is the unique solution to the differential equation $(d / d t) w(t)=i w(t) \alpha(t) h$, $w(0)=1$, where $-\left.i(d / d t) w(t)\right|_{t=0}=h=h^{*} \in A$. Hence, we get $(*)$ and $\gamma$ is the generator of $\alpha$.

Conversely, assume that $\gamma$ is the generator of a group $\alpha$ of $*$-automorphisms on $A$. Let $w$ be the solution to $(d / d t) w(t)=i w(t) \alpha(t)(h), w(0)=1$; then $\varrho(t) a=v^{*} w(t) \alpha(t)(v a)$ is a strongly continuous one-parameter group of isometries on $A$. It is easy to see that the generator $\delta$ of $\varrho$ is determined by (*). This completes the proof.

Remark 3.2. (a) The decomposition of $\delta$ is unique in the sense that $\gamma$ is the generator of the positive part of $\exp (t \delta)$ and for each unitary $v^{*}$ in $\mathscr{D}(\delta)$, there exists exactly one selfadjoint $h$ in $A$ such that $(\delta, \gamma, v, h)$ satisfy (*). In fact, $h=-i v \delta\left(v^{*}\right)$.

If $\left(v^{*}, w^{*}\right)$ is a pair of unitaries in $\mathscr{D}(\delta)$ and $(h, k)$ are the corresponding selfadjoint elements of $A$, i.e. $(v, h)$ and $(w, k)$ satisfy $(*)$, then $i(k-h)$ $=\gamma\left(v w^{*}\right)$.

(b) The above theorem is the infinitesimal version of the fact (Theorem 1.6) that all strongly continuous one-parameter groups $\varrho$ of isometries on a unital $C^{*}$-algebra $A$ are of the form $\varrho(t)=L(u(t)) \alpha(t)$, where $\alpha$ is a strongly continuous group of *-automorphisms on $A$ and $u$ is a continuous unitary 1-cocycle w.r.t. $\alpha$.

COROLlARY 3.3. Let $\delta$ be the generator of a strongly continuous oneparameter group of isometries on a unital $C^{*}$-algebra. If 1 is in $\mathscr{D}(\delta)$, then $\mathscr{D}(\delta)$ is a *-subalgebra of $A, \delta(1)^{*}=-\delta(1)$ and $\gamma=\delta-L(\delta(1))$ is a *derivation. 
Proof. Let $h$ be the selfadjoint element of $A$ corresponding to the unitary $1 \in \mathscr{D}(\delta)$. Then $i h=(d / d t) u(0)=\delta(1)$, hence $\delta(1)^{*}=-i h^{*}=-\delta(1)$. The other statements are part of Theorem 3.1.

Our next result gives an algebraic characterization of the linear maps $\delta$ for which $\delta-L(\delta(1))$ is a $*$-derivation.

Proposition 3.4. Let $A$ be a $C^{*}$-algebra and let $\delta$ be a linear map on $A$ whose domain $\mathscr{D}(\delta)$ is a *-algebra. Fix $h$ in $A$ and let $\gamma=\delta-L(h)$. The following two conditions are equivalent:

(1) (a) $\delta\left(a^{*}\right)=\delta(a)^{*}+h a^{*}-a^{*} h^{*}$

(b) $\delta(a b)=\delta(a) b+a \delta(b)-a h b$.

(2) $\gamma$ is a *-derivation.

Further, if $\delta$ satisfies (1), if $A$ has a unit and $1 \in \mathscr{D}(\delta)$, then $h=\delta(1)$.

Proof. It is easy to see that $\delta$ satisfies 1(a) (resp. 1(b)) if and only if $\gamma$ is symmetric (resp. a derivation)

If $1 \in \mathscr{D}(\delta)$ then $0=\gamma(1)=\delta(1)-h$, i.e. $h=\delta(1)$. The proof is complete.

Corollary 3.5. Let $(\delta, h)$ satisfy (1) of Proposition 3.4, let $\omega$ be a state on $A$ and let $(\mathscr{H}, \pi, \Omega)$ be the cyclic representation associated with $\omega$. If $\mathscr{D}(\delta)$ is dense in $A$ and

$$
\omega(\delta(a))=\omega(h a)
$$

for $a$ in $\mathscr{D}(\delta)$, then there exists a symmetric operator $H$ on $\mathscr{H}$ such that

$$
\mathscr{D}(H)=\pi(\mathscr{D}(\delta)) \Omega, \quad \pi(\delta(a)) f=i((H-i h) \pi(a)-\pi(a) H) f
$$

for $a$ in $\mathscr{D}(\delta)$ and $f$ in $\mathscr{D}(H)$.

Further, $\delta$ is closable if $\pi$ is faithful.

Proof. By assumption $\gamma=\delta-L(h)$ is a $*$-derivation and $\omega(\gamma(a))=0$ for $a$ in $\mathscr{D}(\gamma)$. By [3, Corollary 3.2.27], $\delta$ is closable if $\pi$ is faithful. By [3, Proposition 3.2.28] there exists a symmetric operator $H$ on $\mathscr{H}$ such that

$$
\pi(\gamma(a)) f=i(H \pi(a)-\pi(a) H) t
$$

for $a$ in $\mathscr{D}(\gamma)$ and $f$ in $\mathscr{D}(H)=\pi(\mathscr{D}(\gamma)) \Omega$. From this the proof is easily completed.

Remark 3.6. The study of linear maps $\delta$ satisfying the conclusions of Corollary 3.5 was proposed in [14], where a set of sufficient conditions are stated. These conditions are very strong, in fact, they imply that $\delta$ is a *derivation (i.e. $h=0$ ).

The next corollary should be compared with [13], where a similar result is obtained for a strongly continuous one-parameter group of 2-positive maps.
Corollary 3.7. Let $G$ be a simply connected Lie group and let $X_{1}, \ldots, X_{d}$ be a hasis for the Lie algebra of $G$. Let $\varrho$ be a strongly continuous isometric representation of $G$ on a unital $C^{*}$-algebra and let $\delta_{j}$ be the generator of $t \rightarrow \varrho\left(\exp t X_{j}\right)$ for all $j$. If $1 \in \mathscr{Q}\left(\delta_{j}\right)$ for all $j$, then the following four conditions are equivalent:

(1) Each $\delta_{j}$ is a *-derivation.

(2) $\delta_{j}(1)=0$ for all $j$.

(3) $\varrho(g)$ is a *-automorphism for all $g$ in $G$.

(4) $\varrho(g) 1=1$ for all $g$ in $G$.

Proof. $(1) \Leftrightarrow(2)$ by Corollary 3.3 .

$(3) \Leftrightarrow(4)$ by the uniqueness of the polar decomposition.

(4) $\Rightarrow(2)$ is trivial

$(2) \Rightarrow(4)$. Choose coordinates of the second kind

$$
g=\exp \left(t_{1}(g) X_{1}\right) \ldots \exp \left(t_{d}(g) X_{d}\right)
$$

in a neighbourhood of the unit $e$ in $G$. Then

$$
\varrho(g)=\exp \left(t_{1}(g) \delta_{1}\right) \ldots \exp \left(t_{d}(g) \delta_{d}\right) \text {. }
$$

Hence $\varrho$ has the wanted property in a neighbourhood of $e$ and therefore everywhere [7]. The proof is complete.

THEOREM 3.8. Let $\varrho$ be a strongly continuous isometric one-parameter group on a unital $C^{*}$-algebra, let $(u, \alpha)$ be the polar decomposition of $\varrho$ and let $\delta$ be the generator of $\varrho$. The following eight conditions are equivalent:

(1) $u(s) u(t)=u(s+t)$ for all $s$ and $t$.

(2) $u(s)=\alpha(t) u(s)$ for all $s$ and $t$.

(3) $L(u(t)) \alpha(t)=\alpha(t) L(u(t))$ for all $t$.

(4) $1 \in \mathscr{D}(\delta)$ and $u(t)=\exp (t \delta(1))$.

(5) $1 \in \mathscr{D}(\delta)$ and $\delta(1) u(s)=\alpha(t)(\delta(1) u(s))$ for all $s$ and $t$

(6) $1 \in \mathscr{D}(\delta)$ and $\delta(1)=\alpha(t) \delta(1)$ for all $t$.

(7) $u(s) \in \mathscr{D}(\delta)$ and $\delta(u(s))=\delta(1) u(s)$ for all $s$.

(8) $1 \in \mathscr{T}(\delta), \delta(1) \in \mathscr{T}(\delta)$ and $\delta^{2}(1)=\delta(1)^{2}$.

Proof. (1) $\Leftrightarrow(2) \Leftrightarrow(3) \Leftrightarrow(4)$ by Proposition 2.2 and (the proof of) Theorem 2.5. $(5) \Rightarrow(6)$ is trivial.

$(2) \Leftrightarrow(7)$ and $(6) \Leftrightarrow(8)$ since $\gamma=\delta-L(\delta(1))$ is the generator of $\alpha$ by Corollary 3.3 .

$(2) \Rightarrow(5)$. Since (2) implies (1) and (4) we see that

$$
\begin{aligned}
0 & =\frac{d}{d t} u(s)=\frac{d}{d t} \alpha(t) u(s)=\frac{d}{d t} u(-t) \varrho(t) u(s) \\
& =u(-t) \varrho(t) \delta(u(s))-\delta(u(-t)) \varrho(t) u(s) \\
& =\alpha(t)(\delta(1) u(s))-\delta(1) u(s)
\end{aligned}
$$


$(6) \Rightarrow(4)$. Since

$$
\frac{d}{d t} u(t)=\varrho(t) \delta(1)=R(\delta(1)) u(t)
$$

the uniqueness theorem from semigroup theory [ 8, Theorem 1.7] implies

$$
u(t)=\exp (t R(\delta(1))) u(0)=\sum_{n=0}^{\infty} t^{n} \delta(1)^{n} / n !=\exp (t \delta(1)) .
$$

This completes the proof.

The equivalence $(1) \Leftrightarrow(8)$ in Theorem 3.8 can be formulated as follows:

COROLlaRY 3.9. The one-parameter group $\exp (t \delta)$ is quasi-normal if and only if we may take $v=1$ in Theorem 3.1 and the corresponding $h=h^{*}$ satisfies $h \in \mathscr{D}(\gamma)$ and $\gamma(h)=0$.

It is not likely that one may take $v \neq 1$ in Corollary 3.9.

Remark 3.10. Note that [5, Lemma 2.1] is a trivial consequence of Corollary 3.3, because if $\varrho$ is constructed as in Remark 1.8, then the assumption in [5, Lemma 2.1$]$ is $1 \in \mathscr{D}(\delta)$.

$\$$ 4. Implemented groups. In this section we consider operators on (subalgebras of) $B(\mathscr{H})$ of the form $a \rightarrow i(H a-a K)$, where $H$ and $K$ are operators on the Hilbert space $\mathscr{H}$. Our first result shows that the generator of a norm-continuous one-parameter group of isometries is of this form, with $H$ and $K$ bounded and selfadjoint.

THEOREM 4.1. Let $G$ be a connected locally compact abelian group or a simply connected Lie group. If $\varrho$ is a norm-continuous isometric representation of $G$ on a unital $C^{*}$-algebra $A$, then there exist two norm-continuous unitary representations $U$ and $V$ of $G$ in $A^{\prime \prime}$ (the enveloping von Neumann algebra of $A$ ) such that

$$
\varrho(g) a=U(g) a V(g)^{*}
$$

for all $g$ in $G$ and $a$ in $A$.

Proof. Let $(u, \alpha)$ be the polar decomposition of $\varrho$. By Theorem $1.6, \alpha$ is a norm-continuous representation of $G$ on $A$ by *-automorphisms, hence [9], [24, Theorem 8.5.2] there exists a norm-continuous unitary representation $V$ of $G$ in $A^{\prime \prime}$ such that $\alpha(g) a=V(g) a V(g)^{*}$. Let $U(g)=u(g) V(g)^{*}$. Applying Lemma 1.5 one proves that $U$ is a representation of $G$. This completes the proof.

Remark 4.2. If $G$ is abelian then $g \rightarrow V(g)^{*}$ is a representation of $G$.
Let $\varrho$ be a strongly continuous one-parameter group of isometries on a unital $C^{*}$-algebra $A$, and let $(u, \alpha)$ be the polar decomposition of $\varrho$. By [21, Theorem A1], $A$ may be represented on a Hilbert space $\mathscr{H}$ so that $\alpha$ is convariant, i.e. there exists a strongly continuous unitary group $V$ on $\mathscr{H}$ such that $\alpha(t) a=V(t)^{*} a V(t)$. Let $U(t)=u(t) V(t)^{*}$. Then $\varrho(t) a=U(t) a V(t)$, where $U$ and $V$ are strongly continuous unitary groups on $\mathscr{H}$. If $i H$ (resp. $i K$ ) denotes the generator of $U$ (resp. $V$ ), then there exists a unitary operator $\varphi$ and a selfadjoint operator $h$ on $\mathscr{H}$ such that $\mathscr{D}(K)=\varphi \mathscr{D}(H)$ and $K f$ $=\varphi H \varphi^{*} f+h f$ for $f$ in $\mathscr{D}(K)$ (cf. e.g. [6, Theorem 4.3]). The following discussion is motivated by the observations above.

Definition 4.3. Let $S$ and $T$ be two densely defined unbounded linear operators on $\mathscr{H}$. Denote by $\delta_{S, T}$ the linear map on $B(\mathscr{H})$ defined by $\mathscr{D}\left(\delta_{S, T}\right)$ $=\{a \in B(\mathscr{H}) \mid a \mathscr{D}(T) \subseteq \mathscr{D}(S)$ and $f \in \mathscr{D}(T) \rightarrow(S a-a T) f$ is bounded $\}$ and

$$
\delta_{S, T}(a) f=i(S a-a T) f
$$

for $f$ in $\mathscr{D}(T)$ and $a$ in $\mathscr{D}\left(\delta_{S, T}\right)$. Let $\mathscr{D}_{S, T}$ be the set of $a$ in $B(\mathscr{H})$ for which $(f, g) \in \mathscr{D}(T) \times \mathscr{D}\left(S^{*}\right) \rightarrow\left(a f, S^{*} g\right)-(a T f, g)$ is bounded.

Lemma 4.4. If $S$ is closable with closure $\bar{S}$, then $\mathscr{D}_{S, T}$ equals $\mathscr{D}\left(\delta_{\bar{S}, T}\right)$.

Proof. Fix $a$ in $\mathscr{D}_{S, T}$ and $f$ in $\mathscr{D}(T)$. By assumption, $g \in \mathscr{D}\left(S^{*}\right)$ $\rightarrow\left(a f, S^{*} g\right)$ is bounded, hence $a f \in \mathscr{D}(\bar{S})$ and therefore $a \in \mathscr{D}\left(\delta_{\bar{S}, T}\right)$. The converse inclusion is obvious.

Similarly to the proof of [3, Proposition 3.2.55] one proves

THEOREM 4.5. Let $\exp (i t H)$ and $\exp (-i t K)$ be two strongly continuous semigroups on the Hilbert space $\mathscr{H}$, and let $\eta$ be the weak* continuous semigroup on $B(\mathscr{H})$ defined by

$$
\eta(t) a=\exp (i t H) a \exp (-i t K)
$$

for $a$ in $B(\mathscr{H})$ and $t \geqslant 0$. The generator of $\eta$ is $\delta_{H, K}$.

Corollary 4.6. Let $A \subseteq B(\mathscr{H})$ be a $C^{*}$-algebra and let $\varrho$ be a strongly continuous semigroup on $A$. If $\varrho(t) a=\eta(t) a$ for all $a$ in $A$ and $t \geqslant 0$, then the restriction $\delta_{A}$ of $\delta_{H, K}$ to the set of $a$ in $\mathscr{D}_{H, K} \cap A$ for which $\delta_{H, K}(a) \in A$ is the generator of $\varrho$.

Proof. Let $\delta$ be the generator of $\varrho$ and let $\tilde{\delta}=\delta_{H, K}$. It is obvious that $\delta \subseteq \delta_{A} \subseteq \tilde{\delta}$. Conversely, if $a \in \mathscr{D}\left(\delta_{A}\right)$, then $(1-\tilde{\delta}) a \in A$, hence if $b=$ $(1-\delta)^{-1}(1-\tilde{\delta}) a$, then

$$
(1-\tilde{\delta}) b=(1-\delta) b=(1-\tilde{\delta}) a
$$

and therefore $a=b \in \mathscr{D}(\delta)$. This completes the proof. 
Proposition 4.7. If $S$ is closed, then $\delta_{S, T}$ is both norm-norm and strongstrong closed, and further

$$
\left\{a \in B(\mathscr{H}) \mid \operatorname{ran} a \subseteq \mathscr{D}(S), \operatorname{ran} a^{*} \subseteq \mathscr{D}\left(T^{*}\right)\right\} \subseteq \mathscr{D}\left(\delta_{S, T}\right) .
$$

Proof. Only the last assertion needs a proof. Fix $g$ in $\mathscr{D}\left(S^{*}\right)$ with $\|g\| \leqslant 1$, and let $T_{g}(f)=\left(a f, S^{*} g\right)$ for $f$ in $\mathscr{H}$. Since $\left|T_{g}(f)\right| \leqslant\|S a f\|$, the Banach-Steinhaus Theorem implies $\left|\left(a f, S^{*} g\right)\right| \leqslant M\|f\|\|g\|$ for $f$ in $\mathscr{H}, g$ in $\mathscr{D}\left(S^{*}\right)$ and some fixed $M \geqslant 0$. Likewise $|(a T f, g)| \leqslant M\|f\|\|g\|$ for $f$ in $\mathscr{D}(T)$ and $g$ in $\mathscr{H}$. An application of Lemma 4.4 completes the proof.

THEOREM 4.8. Let $T$ be a densely defined linear map on a Hilbert space and let $\delta=\delta_{T, T^{*}}$

(a) $\delta$ is a derivation if $T$ is symmetric.

(b) $\delta$ is symmetric if $T$ is closed.

(c) $T$ is symmetric if $\delta$ is a $*$-derivation and $T$ is closed.

In particular, if $T$ is closed, then $T$ is symmetric if and only if $\delta_{T, T^{*}}$ is a *-derivation.

Proof. (a) Fix $a$ and $b$ in $\mathscr{D}(\delta)$ and $f$ in $\mathscr{D}\left(T^{*}\right)$. We have $a b \mathscr{D}\left(T^{*}\right) \subseteq a \mathscr{D}(T) \subseteq \mathscr{D}(T)$ and

$$
i\left(T a b-a b T^{*}\right) f=i T a b f+a(\delta(b)-i T b) f=\delta(a) b f+a \delta(b) f .
$$

(b) If $f$ and $g$ are in $\mathscr{D}\left(T^{*}\right)$ and $a$ is in $\mathscr{D}(\delta)$, then the formula

$$
\left(a^{*} f, T^{*} g\right)-\left(a^{*} T^{*} f, g\right)=\left(f, a T^{*} g\right)-\left(T^{*} f, a g\right)
$$

shows $a^{*} \in \mathscr{D}(\delta)$ and $\delta\left(a^{*}\right)=\delta(a)^{*}$.

(c) Fix $a$ and $b$ in $\mathscr{D}(\delta)$ and let $f$ and $g$ be in $\mathscr{D}\left(T^{*}\right)$. Since $\delta$ is a *derivation we get

$(T a b f, g)-\left(a b T^{*} f, g\right)=(a T b f, g)-\left(a b T^{*} f, g\right)-\left(b f, T a^{*} g\right)+\left(b f, a^{*} T^{*} g\right)$.

Hence $(a T b f, g)=\left(b f, T a^{*} g\right)$, and therefore $b f$ is in $\mathscr{D}\left(T^{*}\right)$ and $\left(T b f, a^{*} g\right)$ $=\left(T^{*} b f, a^{*} g\right)$. Choosing $a$ and $b$ suitably one gets $Z(T) \subseteq \mathcal{Y}\left(T^{*}\right)$ and $T f=T^{*} f$ for $f$ in $\mathscr{L}(T)$. Assertions (a) through (c) combined prove the last assertion of the theorem.

Finally, we briefly discuss the extension problem for the *-derivations $\delta_{S}=\delta_{S, S^{*}}$. Trivially $S \subseteq T$ implies $\delta_{S} \subseteq \delta_{T}$. Hence, if $S$ is symmetric and $T$ is a maximal symmetric extension of $S$, then either $\delta_{T}$ or $-\delta_{T}=\delta_{-T}$ is the generator of a weak* continuous semigroup $\alpha$ of $*$-homomorphisms on $B(\mathscr{H})$. It is clear that $\alpha$ cannot be extended to a one-parameter group unless $T$ is selfadjoint. Note that $\delta_{S} \subseteq \operatorname{ad} S \subseteq \delta_{S^{\star}}$; this gives a connection with the extension problem as formulated in [4], [16] and [17].

\section{References}

[1] H. Araki, Expansionals in Banach algebras, Ann. Sci. École Norm. Sup. 6 (1973), 67-84.

[2] E. Berkson, R. J. Flemming and J. Jamison, Groups of isometries on certain ideals of Hilhert space operators, Math. Ann. 220 (1976), 151-156.

[3] O. Bratteli and D. W. Robinson, Operator Algebras and Quantum Statistical Mechanics, Springer, Berlin 1979.

[4] $\mathrm{O} . \mathrm{Bratteli}$ and P. E. T. J $\phi$ rgensen, Unbounded *-derivations and infinitesimal generations on operator algebras, in: Operator algebras and applications (Kingston 1980), Proc. Sympos. Pure Math. 38, part 2, Amer. Math. Soc., Providence, R. I., 1982, 353-365.

[5] - , - Derivations commuting with abelian gauge actions on lattice systems, Comm. Math. Phys. 87 (1982), 353-364.

[6] D. Buchholz and J. E. Roberts, Bounded perturbations of dynamics, ibid. 49 (1976), 161-177.

[7] C. Chevalley, Theory of Lie Groups, Princeton Univ. Press, Princeton 1946.

[8] E. B. Davies, One-Parameter Semigroups, Academic Press, London 1980.

[9] J. Dix mier, Sur les groupes d'automorphismes normiquement continus des $C^{*}$-algèbres, C.R. Acad. Sci. Paris 269 (1969), 643-644.

[10] G. A. Elliot, Convergence of automorphisms in certain $C^{*}$-algebras, J. Funct. Anal. 11 (1972), 204-206.

[11] M. Embry-Wardrop, The partially isometric factor of a semigroup, Indiana Univ. Math. J. 32 (1983), 893-901.

[12] -, Semi-groups of quasinormal operators, Pacific J. Math. 101 (1982), 103-113.

[13] D. E. Evans, Positive linear maps on operator algebras, Comm. Math. Phys. 48 (1976), 1522.

[14] D. P. K. Ghikas, Bi-representations and semi-groups, Lett. Math. Phys. 6 (1982), 253-259.

[15] R. S. Ingarden and A. Kossak owski, On the connection of non-equilibrium information thermodynamics with non-Hamiltonian quantum mechanics of open systems, Ann. Physics 89 (1975), 451-485.

[16] P. E. T. J $\phi$ rgensen, Commutators of Hamiltonian operators and non-abelian algebras, J. Math. Anal. Appl. 73 (1980), 115-133.

[17] -, Extension of unbounded ${ }^{*}$-derivations in UHF $C^{*}$-algebras, J. Funct. Anal. 45 (1982), 341-356.

[18] R. V. Kadison, Isometries of operator algebras, Ann. of Math. 54 (1951), 325-338.

[19] -, Transformation of states in operator theory and dynamics, Topology 3, suppl. 2 (1965), 177-198.

[20] R. R. Kallman, One-parameter groups of *-automorphisms of $\mathrm{II}_{1}$ von Neumann algebras, Proc. Amer. Math. Soc. 24 (1970), 336-340.

[21] A. Kishimoto and D. W. Robinson, On unbounded derivations commuting with a compact group of *-automorphisms, Publ. Res. Inst. Math. Sci. Kyoto Univ. 18 (1982), $1121-1136$.

[22] A. Kossakowski, On quantum statistical mechanics of non-Hamiltonian systems, Rep. Math. Phys. 3 (1972), 247-274.

[23] L. Kristensen, Invariant metrics in coset spaces, Math. Scand. 6 (1958), 33-36.

[24] G. K. Pedersen, $C^{*}$-Algebras and their Automorphism Groups, London Math. Soc. Monographs 14, Academic Press, London 1979.

[25] A. Posiewnik, A sufficient condition for the Hamiltonian evolution, Rep. Math. Phys. 10 (1976), 185-188 
[26] B. Russo and H. A. Dye, $A$ note on unitary operators in $C^{*}$-algebras, Duke Math. J. 33 (1966), 413-416

[27] M. Takesaki, Theory of Operator Algebras I, Springer, New York 1979.

MATHEMATICS INSTITUTE

AARHUS UNIVERSITY

Received October 1, 1986

Revised version April 22, 1987
Added in proof (January 1988). After having finished the work on this paper, the author learned that the norm-continuous case of Theorem 3.1 was handled in: A. M. Sinclair, Jordan homomorphisms and derivations on semisimple Banach algebras, Proc. Amer. Math. Soc. 24 (1970) 209-214.

\section{The universal right $K$-property for some interpolation spaces}

by

MIECZYSŁAW MASTYŁO (Poznań)

Abstract. Under some conditions on a Banach couple $\vec{A}$ and the parameter $\Phi$ of the $K$-method we show that the couples $\left(\vec{A}_{L^{\infty}}, \vec{A}_{\Phi}\right),\left(\vec{A}_{\Phi}, \vec{A}_{L_{1 / 3}^{\infty}}\right)$ have the universal right $K$-property if and only if $\Phi=L_{1 / \varphi}^{\infty}$, where $\varphi$ is the fundamental function of the space $\Phi$. These results are used to obtain a characterization of some symmetric spaces $E$ on $(0, \infty)$ such that the couple $\left(E, L^{\infty}\right)$ has the universal right $K$-property. Moreover, it is proved that the couple $\left(L^{1}, E\right)$ does not have that property.

1. Introduction. We recall some notation from interpolation theory (cf. [4], [13]).

A pair $\vec{A}=\left(A_{0}, A_{1}\right)$ of Banach spaces is called a Banach couple if $A_{0}$ and $A_{1}$ are both continuously imbedded in some Hausdorff topological vector space $V$.

For a Banach couple $\vec{A}=\left(A_{0}, A_{1}\right)$ we can form the intersection $A_{0} \cap A_{1}$ and the sum $A_{0}+A_{1}$. They are both Banach spaces in the natural norms $J(1, a ; \vec{A})$ and $K(1, a ; \vec{A})$, respectively, where

$$
\begin{aligned}
& J(t, a ; \vec{A})=\max \left(\|a\|_{A_{0}}, t\|a\|_{A_{1}}\right), \quad a \in A_{0} \cap A_{1}, \\
& K(t, a ; \vec{A})=\inf _{a=a_{0}+a_{1}}\left(\left\|a_{0}\right\|_{A_{0}}+t\left\|a_{1}\right\|_{A_{1}}\right), \quad a \in A_{0}+A_{1},
\end{aligned}
$$

for $t \in \boldsymbol{R}_{+}=(0, \infty)$.

Let a Banach space $A$ be continuously imbedded in $A_{0}+A_{1}$. The space which consists of all limits in $A_{0}+A_{1}$ of bounded sequences in $A$ is called the Gagliardo completion of $A$ with respect to $A_{0}+A_{1}$ and denoted by $A^{\sim}$. The space $A^{\sim}$ is equipped with the norm $\|a\|_{A} \sim=\inf \sup _{n \geqslant 1}\left\|a_{n}\right\|_{A}$, where the infimum is taken over all sequences $\left\{a_{n}\right\}_{n=1}^{\infty}{ }^{\infty}$ bounded in $A$ such that $a_{n} \rightarrow a$ in $A_{0}+A_{1}$. The closure of $A_{0} \cap A_{1} \subset A$ in $A$ is denoted by $A^{0}$.

Let $\vec{A}=\left(A_{0}, A_{1}\right)$ and $\vec{B}=\left(B_{0}, B_{1}\right)$ be two Banach couples. A linear operator acting from $A_{0}+A_{1}$ into $B_{0}+B_{1}$ will be called a linear mapping from the couple $\vec{A}$ into the couple $\vec{B}$, written $T: \vec{A} \rightarrow \vec{B}$, if $T$ maps continuously $A_{i}$ into $B_{i}, i=0,1$. 